\title{
Fluorescence spectroscopic studies on equilibrium dipole-relaxational dynamics of $\mathrm{Na}, \mathrm{K}-\mathrm{ATPase}$ *
}

\author{
Alexander P. Demchenko ${ }^{\mathrm{a}, 1}$, Hans-Jürgen Apell ${ }^{\mathrm{a}}$, Werner Stürmer ${ }^{\mathrm{a}}$ and Brett Feddersen ${ }^{\mathrm{b}}$ \\ a Department of Biology, University of Konstanz, D-78434 Konstanz (Germany) \\ ${ }^{b}$ Max Planck Institute of Biophysical Chemistry, D-37018 Göttingen (Germany)
}

\begin{abstract}
Intramolecular dynamics in $\mathrm{Na}, \mathrm{K}$-ATPase molecules have been studied by ultraviolet fluorescence spectroscopic methods: determination of temperature-dependent shifts in steady-state spectra, site-selective red-edge effects and their temperature dependence, and time-resolved emission decay as a function of excitation and emission wavelengths. The combination of these methods allows the characterization of the dipolar-relaxational mobility in the environment of the tryptophan residues. Our results show that the mean dipolar-relaxational time is of the order of one nanosecond at room temperature. This is much faster than what is usually observed in globular proteins. The fast dynamics of the protein dipoles are rapid enough so that the dipoles are in dielectric equilibrium during the slower ion transfer processes; this may have important functional consequences.
\end{abstract}

Keywords: Protein dynamics; Na,K-ATPase; Ultraviolet fluorescence; Dipolar relaxations; Red-edge effects; Time-resolved spectroscopy

\section{Introduction}

The $\mathrm{Na}, \mathrm{K}-\mathrm{ATP}$ ase is a protein in the cytoplasmic membranes of animal cells that utilizes the free energy of ATP hydrolysis to transport $\mathrm{Na}$

Correspondence to: H.-J. Apell, Department of Biology, University of Konstanz, Postfach 5560-M635, D-78434 Konstanz, Germany.

* Dedicated to the memory of Peter Läuger, who initiated this research.

1 Permanent address: A.V. Palladin Institute of Biochemistry, Academy of Science of Ukraine, Kiev, 252030, Ukraine. and $\mathrm{K}$ ions against the concentration gradients $[1,2]$. The protein consists of a larger $\alpha$-subunit and a smaller $\beta$-subunit with molecular weights of 100,000 and 45,000 daltons, respectively [3]. The ion translocation is associated with a conformational transition between two distinct conformations of the protein, $E_{1}$ and $E_{2}$. The equilibrium between these forms depends upon the state of phosphorylation and the presence of sodium or potassium ions in the medium. The transition between both conformations can be detected by spectroscopic methods, [4-6].

Fluorescence spectroscopy has been extensively used in the studies of protein structure and 
dynamics. The high sensitivity of the tryptophan fluorescence emission is a useful tool to detect properties of its environment $[7,8]$, especially polarity and mobility. This property is the basis of different applications to study intrinsic ultraviolet fluorescence of proteins [7,9]. The fluorescence method can also be adapted conveniently to obtain dynamic information on the fluorophore environment on a nanosecond time range.

Two complementary approaches are employed. One approach is to analyze the dielectric relaxational dynamics, either by measuring the decay directly or by using the excited state life time, $\tau_{F}$, as an intrinsic time marker [10]. The second approach considers the loss of time-correlation in the positions of groups of atoms surrounding the fluorophore $[7,9,11]$ during relaxation processes. The dynamics should lead to the existence of a wide population of substates with different interaction energies even in the well-determined three-dimensional structure of a protein. Site-selective excitation at long wavelength (at the "red-edge") can excite selectively those species of fluorophore plus environment within a protein, which interact most strongly with the environment in the excited state. They possess the smallest energy of electronic transition $[7,9,11]$. For that reason, the spectra are different when excited at the band maximum and at their red edge, and this difference is a function of the extent of relaxation [7]. However, the investigations of different proteins by the latter approach are fragmentary so far [12-14], and no general conclusion can be made about their relaxation rates up to now. In the tetrameric melittin molecule the dipolar relaxation time is about 40 ns [13], for the staphylococcal nuclease it is in the range of $100 \mathrm{ps}$ [14]. However, usually steady-state red-edge effects are observed that indicate relaxation rates slower than nanosecond.

Single reaction steps in the $\mathrm{Na}, \mathrm{K}$-ATPase contributing to charge translocation across the membrane and the conformational transition from $E_{1}$ to $E_{2}$ have been measured to occur in the millisecond time range $[4,6]$. However, charge translocation and conformational changes probably involve elementary processes which involve dynamic polarization of polar groups in the elec- tric field created by the ions. This effect could be achieved by translocations and rotations of protein groups or domains, and these processes occur probably in the nanosecond time range or faster $[15,16]$. The dynamic polarization of protein groups can also follow the process of light absorption by a tryptophan fluorophore. Its dipole moment is changed by 8-9 Debye units almost immediately upon excitation [7]. This driving force creates an inequilibrium electric field that induces dynamic polarization; the surrounding groups of atoms are induced to relax by changing their positions, and this relaxation convolutes with the decay of the excited state to produce time dependent shifts of emission spectra. The observation of the time dependence reveals direct information on the rate of dynamic polarization in proteins by a fluorescence spectroscopic method.

In the present study of Na,K-ATPase we observed temperature-dependent shifts of fluorescence spectra, measured their dependence on excitation wavelength and detected site-photoselection red-edge effects at different temperatures with and without the application of high concentrations of viscogens (sucrose or glycerol). We conclude from these results, in combination with time-resolved fluorescence decay data, that the relaxational dynamics in the protein interior occurs in the nanosecond time range.

\section{Materials and methods}

\subsection{Materials}

Sodium dodecylsulfate (SDS) was obtained from Pierce Chemical (Rockford, IL), sodium cholate from Merck (Darmstadt) and dioleoyllecithin from Avanti Polar Lipids (Birmingham, AL). Phosphoenolpyruvate, pyruvate kinase, lactate dehydrogenase, NADH and ATP (disodium salt, special grade) were from Boehringer (Mannheim). Sucrose, glucose and ethylenediamine tetraacetic acid (EDTA) were purchased from Sigma. In the experiments with nominal absence of $\mathrm{K}^{+}, \mathrm{NaCl}$ was used in Suprapur quality (Merck). All other reagents were analytical grade. 
Sephadex G25 was obtained from Serva (Heidelberg).

\subsection{Enzyme preparation}

$\mathrm{Na}, \mathrm{K}-\mathrm{ATP}$ ase was prepared from outer medulla of rabbit kidneys using procedure $\mathrm{C}$ of Jørgensen [17]. This method yields purified enzyme in the form of membrane fragments containing about $0.8 \mathrm{mg}$ phospholipid and $0.2 \mathrm{mg}$ cholesterol per $\mathrm{mg}$ protein [18]. The specific ATPase activity was determined by the pyruvate kinase/lactate dehydrogenase assay [19] and the protein concentration by the Lowry method [20], using bovine serum albumin as a standard. For most preparations the specific activity was in the range between 1500 and $2200 \mu \mathrm{mol} \mathrm{P}_{\mathrm{i}}$ per hour and $\mathrm{mg}$ protein at $37^{\circ} \mathrm{C}$, corresponding to a turnover rate of $120-170$ $\mathrm{s}^{-1}$ (based on a molar mass of $280,000 \mathrm{~g} / \mathrm{mol}$ ). The suspension of Na,K-ATPase-rich membrane fragments (about $3 \mathrm{mg}$ protein per $\mathrm{ml}$ ) in buffer (25 $\mathrm{m} M$ imidazole sulfate, $\mathrm{pH} 7.5,1 \mathrm{~m} M$ EDTA, $10 \mathrm{mg} / \mathrm{ml}$ saccharose) was frozen in samples of $100 \mu \mathrm{l}$; in this form the preparation could be stored for several months at $-70^{\circ} \mathrm{C}$ without significant loss of activity. Enzyme concentration in solutions used for fluorescence experiments was typically $0.2 \mathrm{mg} / \mathrm{ml}$.

\subsection{Reconstituted vesicles}

Reconstituted vesicles with membrane-incorporated $\mathrm{Na}, \mathrm{K}$-ATPase were prepared as described previously [21]. Na,K-ATPase membranes were solubilized in sodium cholate. After addition of dioleoyl phosphatidylcholine in sodium cholate, the detergent was removed by dialysis at $4^{\circ} \mathrm{C}$ for $60 \mathrm{~h}$. This yields unilamellar vesicles with an average diameter of $96 \mathrm{~nm}$ [21].

\subsection{Buffers}

All the experiments were performed in $30 \mathrm{mM}$ imidazole buffer, $\mathrm{pH}$ 7.2, containing $1 \mathrm{mM}$ EDTA. To obtain the enzyme preferentially in the $\mathrm{E}_{1}$ or $\mathrm{E}_{2}$ form $50 \mathrm{mM}$ of $\mathrm{NaCl}$ or $\mathrm{KCl}$ were added respectively.

\subsection{Enzyme solutions in the presence of viscogens}

The buffered solutions of enzyme in the presence of high concentrations of sucrose $(44 \%)$ and glycerol $(57 \%)$ were prepared before the measurements. The results were reproducible after storage overnight. After storage no decline of the enzyme activity was detected in the standard activity test.

\subsection{Fluorescence measurements}

The studies of fluorescence spectra at low temperatures were performed with a Hitachi MPF-4 spectrofluorimeter equipped with a quartz dewar. The temperature was maintained by a homemade device producing flow of cold nitrogen vapor and measured with a thermocouple. The sample was immersed in a quartz tube of $3 \mathrm{~mm}$ in diameter. To reduce the stray light an additional cut-off filter was used in the excitation beam. The slits of the monochromators for excitation and emission wavelengths were set 3 and $5 \mathrm{~nm}$ respectively.

At temperatures above $0^{\circ} \mathrm{C}$ the excitationwavelength dependence of fluorescence spectra was studied with a Perkin-Elmer $650-40$ fluorescence spectrophotometer, equipped with a thermostated cuvette holder. The slits were set to 2 $\mathrm{nm}$ for excitation and $5 \mathrm{~nm}$ for emission.

\subsection{Time-resolved spectroscopy}

Time-resolved data on fluorescence-emission decay were obtained on a frequency-domain fluorometer equipped with wide-band acousto-optic light modulator in a frequency range of 20 to 200 $\mathrm{MHz}$, constructed in the Max-Planck-Institute of Biophysical Chemistry in Göttingen, Germany [22]. The sample was excited by a Spectra-Physics 2035 argon-ion laser through interference filters (bandwidth $10 \mathrm{~nm}$ ) with transmission maxima at 290 or $300 \mathrm{~nm}$. The latter filters caused a transmission of different proportions the laser lines at 301 and $305 \mathrm{~nm}$. Emission wavelengths were selected by interference filters with a bandwidth of $10 \mathrm{~nm}$. The protein samples were the same as for steady-state fluorescence studies, the temperature was set to $20^{\circ} \mathrm{C}$. 


\section{Results}

\subsection{Temperature dependent studies of fluorescence spectra}

The position of maximum fluorescence emission of tryptophan in proteins depends upon interactions of the chromophores with specific groups in the local environment. In general, they are controlled by two different universal factors: polarity and dynamics of the environment of a tryptophan residue [7-9]. Polarity refers to the magnitudes and concentrations of the surrounding dipoles, and dynamics concerns the rate of their reorientation. If the dipoles have slow reorientation rates compared to the lifetime of the excited state, the observable effect is the same as if they are static. In this case, dipolar interactions are small and they should shift the excitation spectra to higher excitation energies. In order to obtain dynamic information from the spectral shifts one must know the limiting cases-the slow and fast relaxation limits within the same protein conformation. Studies over an extended temperature interval are complicated with protein structures, because they are not stable above their transition temperatures, typically in the range of $40^{\circ} \mathrm{C}$. At temperatures below $0^{\circ} \mathrm{C}$ pure water freezes; this produces significant effects on the properties of dissolved proteins. The addition of glycerol stabilizes the native protein conformation at high temperature [23,24], and prevents the formation of water crystals in the sub-zero temperature range, glycerol-water mixtures form a transparent glass at low temperatures.

In Fig. 1 the temperature dependence of the intrinsic emission spectra $\mathrm{Na}, \mathrm{K}$-ATPase is presented for two different excitation wavelengths (290 $\mathrm{nm}$ and $305 \mathrm{~nm}$ ), for room temperature $\left(24^{\circ} \mathrm{C}\right)$ and low temperature $\left(-100^{\circ} \mathrm{C}\right)$. The aqueous buffer contains $57 \%$ glycerol. When exciting at $290 \mathrm{~nm}$ the emission spectrum is shifted substantially to shorter wavelengths when the temperature is decreased; the shape of the spectrum remains unchanged. When excited at $305 \mathrm{~nm}$ the spectrum shows no temperature-dependent shift. This dependence of the spectral shift upon the wavelength of excitation will be discussed in the next section.

In model experiments measuring the emission spectra of indole or tryptophan in glycerol or propylene glycol solutions $[7,9,10]$ the shift of fluorescence spectra are temperature-dependent with a sigmoidal shape. At the low-temperature limit the dipoles surrounding the fluorophore cannot reorient during the fluorescence emission lifetime. In the high-temperature limit these relaxations are too fast to influence the time dependence of the spectra. The half-transition point, $t_{1 / 2}^{0}$, should correspond to the condition when the relaxation time $\tau_{R}$ is equal to the emission lifetime $\tau_{\mathrm{F}}$.

The results of temperature-dependent studies of the Na,K-ATPase in buffer containing $50 \mathrm{mM}$ $\mathrm{NaCl}$ and $57 \%$ of glycerol demonstrate a more complex behavior than model studies. A single sigmoid function which usually describes the temperature-dependent shifts of fluorescence spectra $[7,26]$ does not result in a good fit to experimental data (Fig. 2). Instead we observe clearly two steps in the temperature dependence of spectra: between 45 and $-10^{\circ} \mathrm{C}$, and between -25 and $-100^{\circ} \mathrm{C}$ (Fig. 2A). The approximation by a superposition of two sigmoid functions, each expressed in the form:

$\lambda_{\max }^{\mathrm{F}}=\frac{\lambda_{\infty}+\lambda_{0}}{2}+\frac{\lambda_{\infty}-\lambda_{0}}{2} \cdot \frac{t^{0}-t_{1 / 2}^{0}}{\sqrt{d^{2}+\left(t^{0}-t_{1 / 2}^{0}\right)^{2}}}$

where $\lambda_{\max }^{\mathrm{F}}$ is the maximum of fluorescence spectrum at temperature $t^{0}$, and $\lambda_{\infty}$ and $\lambda_{0}$ are its values for the high-temperature and low-temperature limits respectively, $t_{1 / 2}^{0}$ is the half-transition point, and $d$ is the transition half-width, resulted in the following: The parameters of the high-temperature transition range are: $\lambda_{\infty}=340.5 \mathrm{~nm}, \lambda_{0}$ $=333.7 \mathrm{~nm}, t_{1 / 2}^{0}=16^{\circ} \mathrm{C}$ and $d=12^{\circ} \mathrm{C}$. The low temperature transition is fitted by $\lambda_{\infty}=334.3 \mathrm{~nm}$, $\lambda_{0}=329.2 \mathrm{~nm}, t_{1 / 2}^{0}=-64^{\circ} \mathrm{C}$ and $d=20^{\circ} \mathrm{C}$.

For comparison a tryptophan solution in $57 \%$ glycerol has been examined in the temperature range between $-100^{\circ} \mathrm{C}$ and $50^{\circ} \mathrm{C}$ (Fig. 2B). A single sigmoidal curve has been found as expected. The wavelength positions $\left(\lambda_{0}=317 \mathrm{~nm}\right.$ 


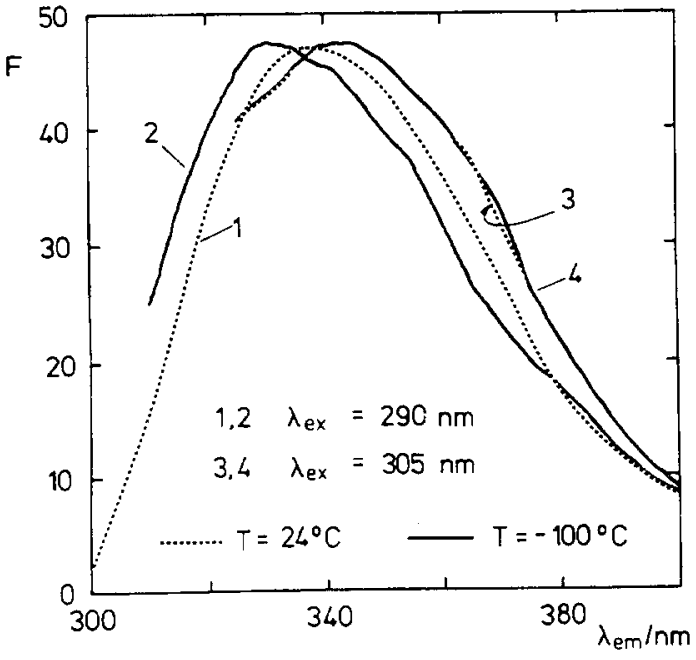

Fig. 1. Fluorescence spectra of Na,K-ATPase in buffer con-

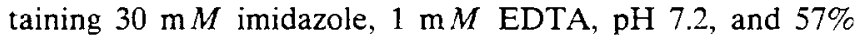
glycerol at $24^{\circ} \mathrm{C}(1,3)$ and $-100^{\circ} \mathrm{C}(2,4)$ at excitation wavelengths $290 \mathrm{~nm}(1,2)$ and $305 \mathrm{~nm}(3,4)$.

and $\lambda_{\infty}=346 \mathrm{~nm}$ ) and the half-transition point $\left(t_{1 / 2}^{0}=-48^{\circ} \mathrm{C}\right)$ lead us to the conclusion, that the lower transition of the Na,K-ATPase data corresponds to those tryptophanes which are closer to

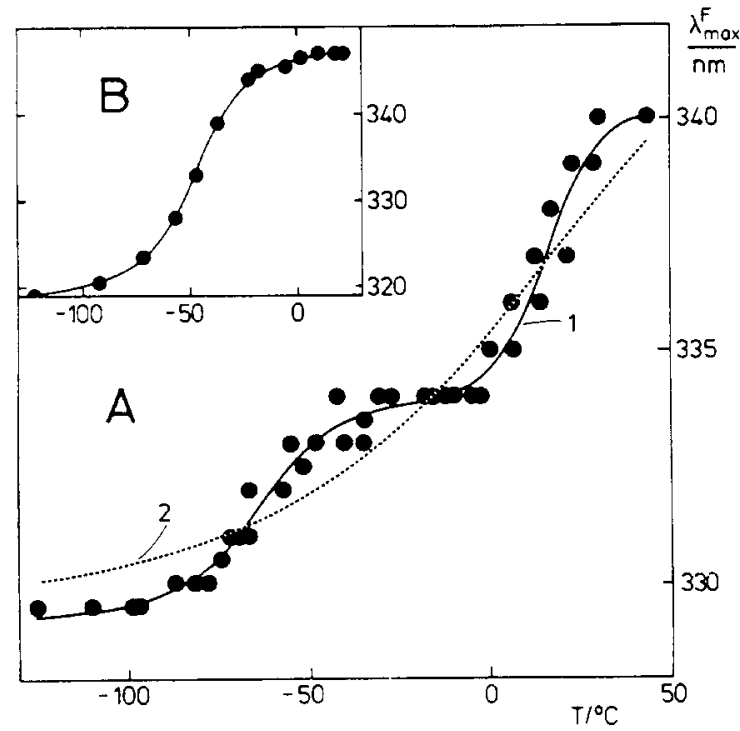

Fig. 2. Temperature dependence of positions of the fluorescence maximum, $\lambda_{\max }$, of the steady-state fluorescence spectra of $\mathrm{Na}, \mathrm{K}-\mathrm{ATPase}$ in buffer as given in Fig. 1 (excitation at $290 \mathrm{~nm}$ ). (A) Results of a typical experiment. (1) - the approximation by a superposition of two sigmoid functions. (2) - the approximation by a single sigmoid function. (B) Temperature dependence of the fluorescence of tryptophan dissolved in water containing $57 \%$ glycerol. the surface and which are under influence of the solvent dynamics.

The higher temperature transition apparently reports upon dielectric relaxations in the $\mathrm{Na}, \mathrm{K}$ ATPase protein, and because the fluorescence emission occurs in the range of nanoseconds (see below), our results suggest that the characteristic time for the protein dielectric relaxations is in the nanosecond time domain. To further investigate this transition we have measured the excitationwavelength dependence of the spectra ("red-edge effect") and a dependence of time-resolved emission decay on excitation and emission wavelengths.

\subsection{Red-edge effect}

The dependence of fluorescence spectra of $\mathrm{Na}, \mathrm{K}$-ATPase on excitation wavelength has been studied. At the maximum to the short wavelength range of the absorption spectrum (i.e. in the range of $270-290 \mathrm{~nm}$ ) no influence on the excitation wavelength was observed. However, an effect was demonstrated clearly at the red edge of the absorption spectrum ( $295 \mathrm{~nm}$ and above); a substantial shift of the maximum of fluorescence spectra to longer wavelengths (Fig. 1, e.g. traces 1 and 3 ) is seen when exciting in this red region of the absorption curve. If this effect is associated with the nanosecond dipolar relaxation of the fluorophore environment, a characteristic pattern is expected, namely the spectra should display temperature dependent maximum wavelength at the main-band excitation, but at the red edge excitation this temperature dependence should vanish (excitation at 305-307 $\mathrm{nm}$ ) [7]. The red excitation photoselects those molecules that have lowest energy of interaction with the environment at any temperature; the conformations of these photoexcited molecules are already in the "relaxed state" with respect to their environment, before absorbing a photon. The results in Fig. 1 (traces 3 and 4) demonstrate that an effect of temperature on the maximum of the fluorescence spectrum of $\mathrm{Na}, \mathrm{K}$-ATPase excited at the red edge could not be observed, and the maximum of fluorescence is substantially shifted to longer wavelengths. 


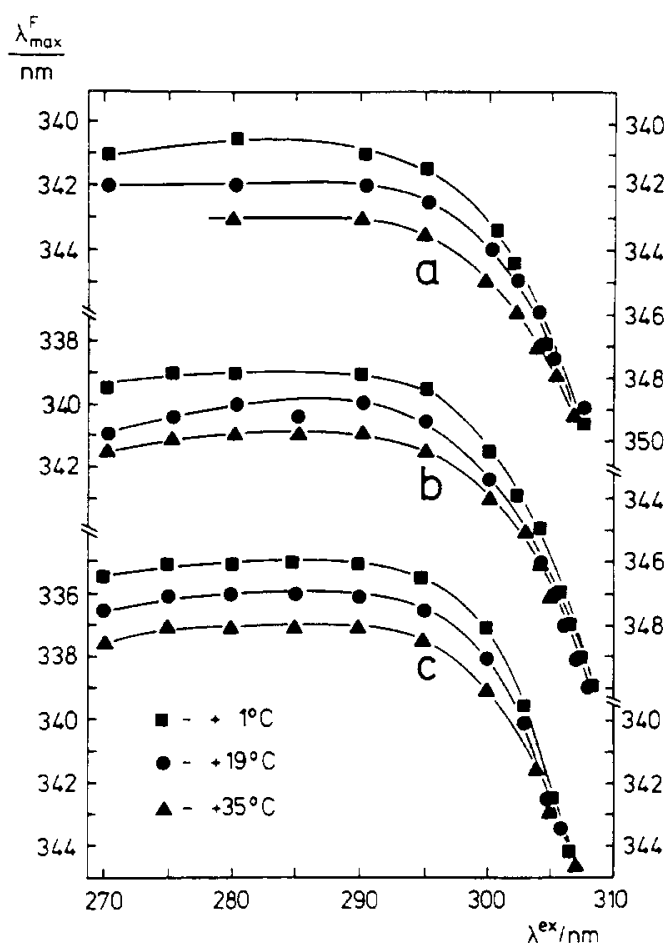

Fig. 3. Excitation wavelength dependence of the position of the maximum, $\lambda_{\max }^{F}$, of fluorescence spectra of Na,K-ATPase in membrane fragments at different temperatures. a: in buffer containing $30 \mathrm{~m} M$ imidazole, $1 \mathrm{~m} M$ EDTA, and $50 \mathrm{~m} M$ $\mathrm{NaCl}, \mathrm{pH} 7.2$, b: additionally $44 \%$ sucrose, c: additionally to a $57 \%$ glycerol.

The dependence of the position of maxima of the fluorescence emission spectra on excitation wavelengths shows a pronounced effect on the red edge, which, as expected, does not tend to any limit (Fig. 3). These experiments have been performed in a conventional spectrofluorometer which allows only temperatures in a range between 1 and $35^{\circ} \mathrm{C}$. We extended our studies to enzyme preparations containing no glycerol and in which glycerol was substituted by another viscogen, sucrose. We observed the same temperature-dependent changes in positions of fluorescence maximum as a function of the excitation wavelength, the results converged and superimposed at 305-307 nm. The excitation wavelength could not be increased further because the intensity became too low to determine a maximum.

It is essential that the observed red-edge effect with and without viscogen was very similar. This means that the viscogen does not influence substantially the dynamics of the protein which is detected by tryptophanes. The small-scale dynamics controlling the dipolar protein relaxations in the temperature range between 1 and $35^{\circ} \mathrm{C}$ are apparently uncoupled with the dynamics of the solvent. The general conclusion drawn from the relaxation rates obtained by the low-temperature studies in the presence of high concentrations of glycerol, can be extended to solutions of enzyme in an aqueous buffer. However, some minor differences, have to be considered. In glycerol and sucrose the red-edge effect tended to be more pronounced. The addition of these viscogens shifted the emission spectra not only when excited at the red edge, but also when excited at absorption maximum. This could be the result of a perturbation of the tryptophan residues by the solvent or of some minor conformational modifi-

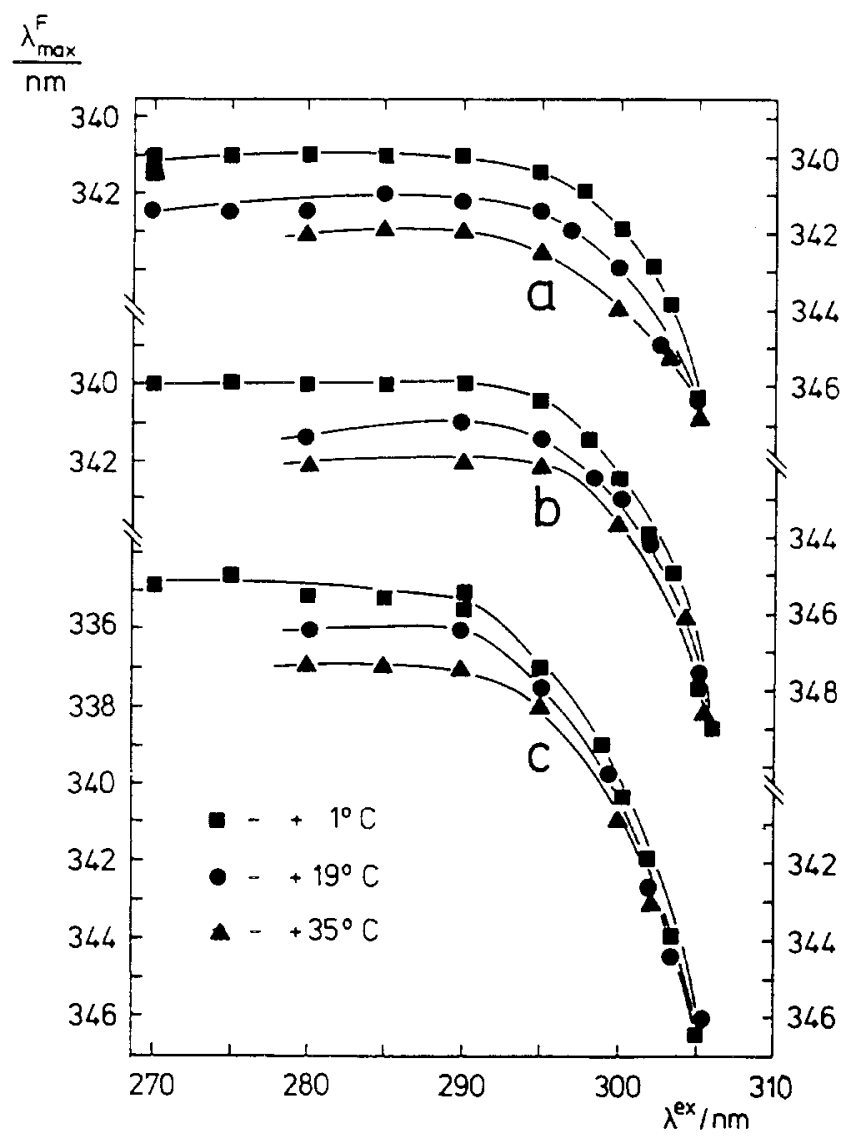

Fig. 4. Excitation wavelength dependence of position of the maximum, $\lambda_{\max }^{F}$, of fluorescence spectra of $\mathrm{Na}, \mathrm{K}$-ATPase, reconstituted into phospholipid vesicles, at different temperatures. a: in buffer containing $30 \mathrm{~m} M$ imidazole, $1 \mathrm{~m} M$ EDTA and $50 \mathrm{mM} \mathrm{NaCl}, \mathrm{pH} 7.2$, b: additionally $44 \%$ sucrose, $\mathrm{c}$ : additionally to a $57 \%$ glycerol. 
cations of the protein, which influence the tryptophanes and/or their environment.

In order to investigate whether the packing and composition of lipids attached to the protein has an influence on the tryptophan fluorescence, we performed a series of experiments with $\mathrm{Na}, \mathrm{K}$ ATPase reconstituted into phospholipid vesicles. The results are presented in Fig. 4. A comparison with the data on native membrane fragments (Fig. 3) demonstrated that the red-edge effect was present, and it displayed essentially the same regularity. Moreover, the position of the fluorescence maxima and the magnitude of the red-edge effect were basically retained. Reconstitution into lipid vesicles did not alter the fluorescence spectra at the main-band excitation in aqueous buffer or in the presence of glycerol, whereas a shift by $2 \mathrm{~nm}$ to shorter wavelength was observed in sucrose containing buffer. In the case of reconstituted enzyme the magnitude of the red-edge effect tended to be increased in glycerol and sucrose. Essentially the same results were found for the temperature dependence of the effect in membrane fragments and vesicles. These findings suggest that the pattern of dynamics we are characterizing reflected intrinsic properties of the ATPase molecule and was not influenced substantially by the surrounding environment.

\subsection{Time-resolved spectroscopic studies}

The results of fluorescence experiments with nanosecond time resolution are presented in Fig. 5 and Table 1. They demonstrate mean excited-state lifetimes, $\tau_{F}$, in the range of $5 \mathrm{~ns}$. For tryptophan in water at neutral $\mathrm{pH}$ an excited-state life time $\tau_{F}$ of 3 ns has been found. In proteins the variation of this parameter is very wide, it ranges from less than $1 \mathrm{~ns}$ to $8 \mathrm{~ns}$ [25]. As is the case for many other proteins [25] a decay function with two exponentials produced a much better fit to the data, compared to a single exponential curve (Fig. 5). A comparison of the time-resolved data excited at $290 \mathrm{~nm}$ with an emission of 340 $\mathrm{nm}$ (at the fluorescence maximum) or $380 \mathrm{~nm}$ (at the long-wavelength slope) demonstrates that increasing the emission wavelength results in a reduction of amplitude and lifetime of the shorter

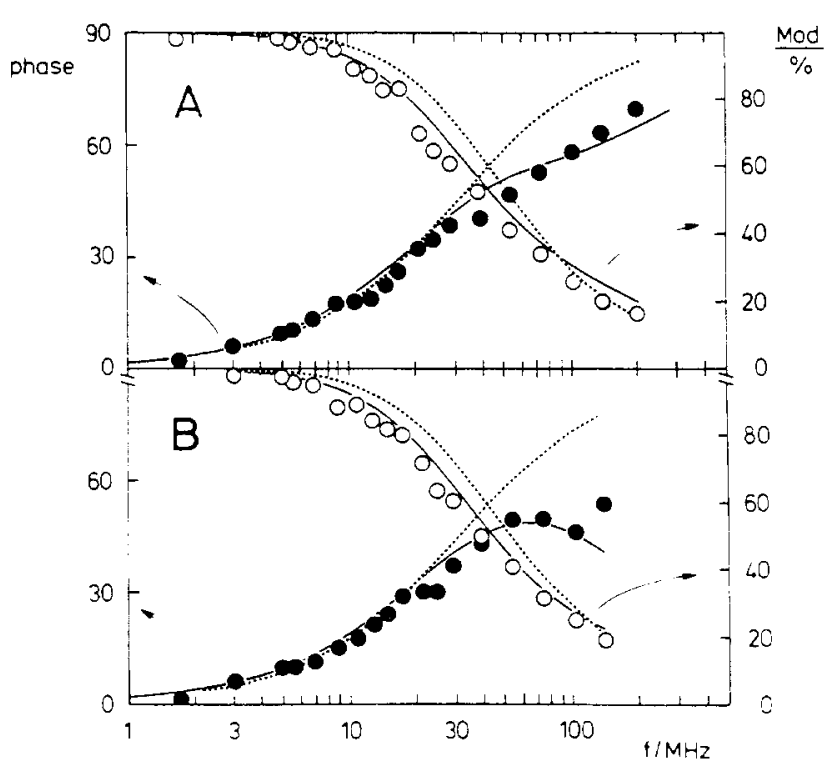

Fig. 5. Frequency dependence of phase and modulation data for ultraviolet fluorescence of Na,K-ATPase. Approximations with single (dashed lines) and double exponential (solid lines) decay functions. Full circles present phase, open circles modulation data. Experimental conditions are (A): Excitation 290 $\mathrm{nm}$, emission $380 \mathrm{~nm}$, bandpass $10 \mathrm{~nm}$, buffer as given in Fig. 1. Experiments were performed at $20^{\circ} \mathrm{C}$. (B): Excitation $300 \mathrm{~nm}$, emission $360 \mathrm{~nm}$, bandpass $10 \mathrm{~nm}$, buffer as in part A. Addition of $57 \%$ glycerol had only minor influence on the experimental data (not shown, cf. Table 1).

component of the fluorescence decay (Tab. 1). This reduction is most probably the result of a nanosecond dipolar relaxation which is known to shift the fluorescence spectrum in time to longer wavelengths $[26,27]$. The relaxation process actually withdraws emission intensity from the short-

Table 1

Results of single and double exponential analysis of emission decay data of Na,K-ATPase (in $\mathrm{K}^{+}$ions containing buffer) at different excitation $\left(\lambda^{\mathrm{ex}}\right)$ and emission $\left(\lambda^{\mathrm{em}}\right)$ wavelengths

\begin{tabular}{lllllll}
\hline $\begin{array}{l}\lambda^{\mathrm{ex}} \\
(\mathrm{nm})\end{array}$ & $\begin{array}{l}\lambda^{\mathrm{em}} \\
(\mathrm{nm})\end{array}$ & $a_{1}$ & $\begin{array}{l}\tau_{1} \\
(\mathrm{~ns})\end{array}$ & $a_{2}$ & $\begin{array}{l}\tau_{2} \\
(\mathrm{~ns})\end{array}$ & $\chi^{2}$ \\
\hline 290 & 340 & 1.000 & 4.880 & - & - & 1.080 \\
& & 0.772 & 6.792 & 0.228 & 1.138 & 0.419 \\
290 & 380 & 1.000 & 5.083 & - & - & 1.141 \\
& & 0.844 & 6.486 & 0.156 & 0.845 & 0.506 \\
300 & 360 & 1.000 & 5.285 & - & - & 2.581 \\
& & 0.856 & 6.590 & 0.144 & 0.000 & 0.494 \\
$300{ }^{\mathrm{a}}$ & 360 & 1.000 & 4.568 & - & - & 1.389 \\
& & 0.888 & 5.491 & 0.112 & 0.286 & 0.654 \\
\hline
\end{tabular}

In $57 \%$ glycerol. 
wavelength part of the spectrum and adds it in the time course of emission to its long-wavelength part. In model media (polar solutions of different dyes) one can even observe at sufficiently long wavelengths a component with negative amplitude; which is due to a non-emissive dynamic process in the excited state. In studies with proteins this effect is very difficult to observe [7]. Two reasons can explain the absence of such observations: (1) In the studied proteins the relaxation rates of the dipoles were outside the nanosecond time interval and (2) the fluorescence decay itself is multiexponential and a short-living negative component due to relaxation is superimposed by the positive one due to heterogeneity in emission. This can be due to the distribution of lifetimes of individual tryptophan residues in the case of multi-tryptophan proteins, or due to the distribution of conformations with different quenching probabilities, which can even occur in single-tryptophan proteins. In the case of $\mathrm{Na}, \mathrm{K}$ ATPase, where the exponential behavior is emission-wavelength dependent the effect can arise from a distribution of lifetimes of the individual tryptophanes, and those, which have longer $\tau_{\mathrm{F}}$, emit at longer wavelengths.

This explanation seems to be in contradiction to the results obtained with an excitation at 300 $\mathrm{nm}$ and an intermediate emission wavelength of $360 \mathrm{~nm}$. Under these conditions we observed a decay of emission which could be described reasonably by a single exponential curve (A small short-living component is probably an artifact due to light-scattering). However, this finding can be explained by the fact that the tryptophanes excited selectively at the red edge have the highest interaction energy with the environment. They are closest to the relaxed state and therefore display essentially a more homogeneous emission. Thus we think that the fluorescence decay that is wavelength dependent is directly related to the relaxation of the environment of the tryptophan occurring in the same time range. In a glycerol containing buffer a comparable observation was made, exciting the tryptophanes at $300 \mathrm{~nm}$. The short-lived component was shorter than 0.3 ns and had an amplitude in the range of $10 \%$ of the total signal. In the case without glycerol the short-lived component could be due to incomplete elimination at light-scattering band. The value of $\tau_{F}\left(=\tau_{1}\right)$, however, is slightly reduced compared to measurements without glycerol (Table 1). The effects of viscogens on the emission decay and on the position of the fluorescence maxima (Fig. 3) could be the result of some minor conformational rearrangements in protein or a perturbation of those tryptophan residues which are in direct contact with the solvent.

\subsection{Differences in $\mathrm{Na}^{+}$or $\mathrm{K}^{+}$-containing buffers}

It is known that the Na,K-ATPase can be forced into two different conformations by changing the ionic composition of the aqueous buffers. In the presence of $\mathrm{Na}^{+}$(and no ATP) the enzyme is in the $E_{1}$ form with up to $3 \mathrm{Na}^{+}$bound to the ion binding sites facing the cytoplasmic interface. In the presence of $\mathrm{K}^{+}$(and absence of $\mathrm{Na}^{+}$and ATP) the enzyme has occluded $2 \mathrm{~K}^{+}$in its $\mathrm{E}_{2}$ form $[1,2]$. Therefore, the fluorescence spectra have been investigated not only in a $50 \mathrm{mM}$ $\mathrm{Na}+$-containing buffer as shown in Fig. 3, but

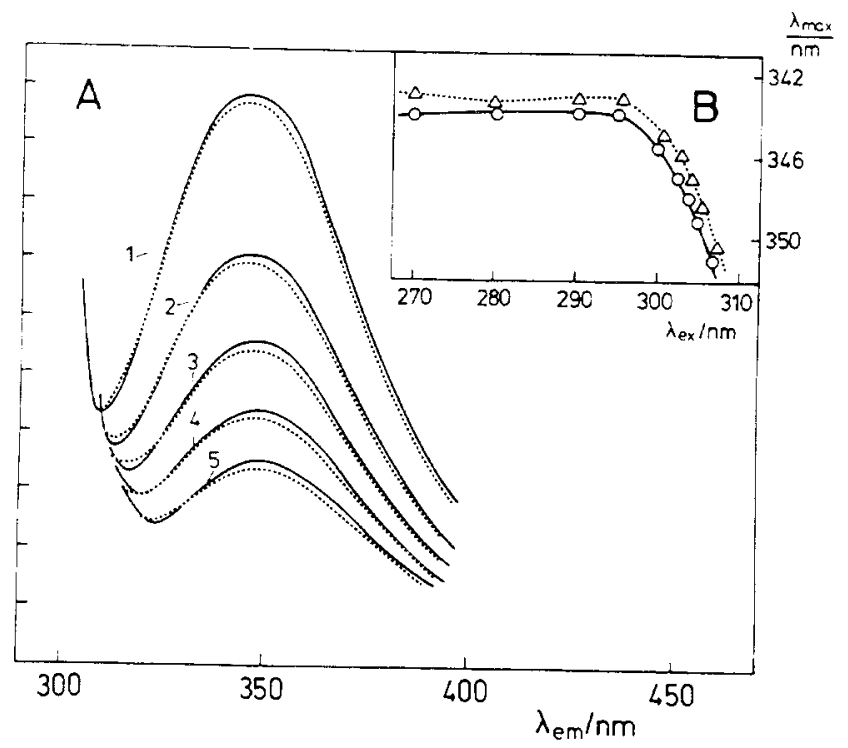

Fig. 6. Influence of cations on the tryptophan fluorescence of membrane fragments containing $\mathrm{Na}, \mathrm{K}$-ATPase in aqueous buffer ( $30 \mathrm{~m} M$ imidazole, $1 \mathrm{~m} M$ EDTA, pH 7.2) and $50 \mathrm{~m} M$ $\mathrm{NaCl}$ (solid lines) or $50 \mathrm{~m} M \mathrm{KCl}$ (dashed lines), temperature $T=18^{\circ} \mathrm{C}$. (A) Fluorescence spectra, (B) Position of the fluorescence maximum. Excitation wavelengths were $300 \mathrm{~nm}(1)$, $302 \mathrm{~nm}(2), 304$ (3), 305 (4) and 307 (5). 
also in a buffer containing $50 \mathrm{mM} \mathrm{K} \mathrm{K}^{+}$instead. The comparison of the data showed a uniform and therefore significant shift of $0.5-1 \mathrm{~nm}$ of the fluorescence maximum to shorter wavelengths in $\mathrm{K}^{+}$-containing buffers (Fig. 6). This shift was independent of temperature (determined at $+1^{\circ} \mathrm{C}$, $19^{\circ} \mathrm{C}$ and $35^{\circ} \mathrm{C}$ ) and excitation wavelength (monitored between $270 \mathrm{~nm}$ and $308 \mathrm{~nm}$ ).

\section{Discussion}

\subsection{Heterogeneity of tryptophan residues}

$\mathrm{Na}, \mathrm{K}$-ATPase is a multitryptophan protein $[28,29]$. The $\alpha, \beta$-protomer of the sheep kidney enzyme contains 16 tryptophanes and its secondary structure has been well investigated $[29,30]$. Four belong to the $\beta$-subunit, none is found in the transmembrane segment, and 10 tryptophanes have been found in the $\alpha$-subunit, with only 3 in the proposed transmembrane segments [30]. The six tryptophanes situated in the portion of the molecule protruding into the cytoplasm may be buried within the protein matrix, or may face the surface to the aqueous environment. Therefore, we have to consider different types of heterogeneity in the environment of these residues.

At first we have to consider position heterogeneity. Since in each protein conformation every tryptophan is surrounded by groups of atoms in particular positions and orientations. This type of heterogeneity is responsible for the variance in wavelengths of the maximum of protein fluorescence spectra, ranging from $307 \mathrm{~nm}$ (azurin) to $350 \mathrm{~nm}$ (peptides and proteins with tryptophan residues exposed to aqueous solvent) [7]. If we assume that there is no excited-state energy transfer between tryptophanes, the fluorescence spectrum of the protein should be the sum of the contributions of the individual tryptophanes, and it can be expected that the spectrum is broader than for a single-tryptophan protein, because the tryptophanes emit at different wavelengths. However, the width at half-maximum intensity of the fluorescence spectrum for Na,K-ATPase is $59 \mathrm{~nm}$ [31], which is not substantially broader than the spectra of single-tryptophan proteins emitting at these wavelengths [32]. According to Burstein's classification the spectrum of an individual tryptophan residue emitting at $340-342 \mathrm{~nm}$ should have a half-width of 53-55 nm [32]. The heterogeneity of the position of the tryptophan in (dog kidney) $\mathrm{Na}, \mathrm{K}$-ATPase has been studied in quenching experiments with acrylamide [33]. In $\mathrm{E}_{1}$ and $\mathrm{E}_{2}$ conformation acrylamide had an access to different pools of tryptophanes, but no shift of fluorescence maximum was observed on quenching [33]. This suggests that the positiondependent differences of tryptophan interactions with their environments are not significant.

The absorption spectrum of individual tryptophanes could also be influenced by the heterogeneity of position, and this effect would influence the photoselection at the red edge. The position of the absorption spectrum, however, is known to be much less affected than the fluorescence spectrum. These shifts are usually in the range of $200-300 \mathrm{~cm}^{-1}$ without substantial change of the shape of the spectrum [7]. If the two forms are present in equal proportions, the largest photoselection should be expected at the point of steepest decline of the spectrum (maximum of the first derivative), which is at 293-295 $\mathrm{nm}$ for tryptophan in proteins [7], and it should decrease on further motion to the red edge, producing a wavelength dependence different from that observed in single-tryptophan proteins. In our experiments, however, the excitation wavelength dependence of the spectra exhibits essentially the same features as in single-tryptophan proteins and model solutions of tryptophan $[12,13]$ : the shift is gradually enlarging with increase of excitation wavelength and the plots taken at different temperatures converge to the same points at 305-307 nm (Figs. 3 and 4). As a consequence the position-determined photoselection of the side is not considered as significant, especially at the far red edge.

A second type of heterogeneity arises from the intramolecular protein dynamics. The surrounding groups change their positions and orientations causing the local environments of tryptophanes to fluctuate. The result is a broadening of both the absorption and emission spectra. If the 
excited fluorophore has a high dipole moment, as in the case of tryptophan [7], this broadening could increase the width of absorption spectra by $300-500 \mathrm{~cm}^{-1}[34,35]$. The most significant contribution to this type of broadening is caused by dipole-dipole interactions [26]. Such distributions are the origin of the red-edge effects [7,9]. Since both, peptide groups and many of the side chains, possess large dipole moments, they contribute to a significant broadening [9].

\subsection{Dipole-relaxational dynamics}

The dynamics of dipole relaxations is the major contribution to time-dependent shifts of fluorescence spectra in polar environments. In liquid solvents dipole motions can be observed in the time ranges of several picoseconds [36,37]. However, if the fluorophore environment becomes highly viscous they are prolonged to nanoseconds or longer [27]. It has been shown that many protein molecules behave in liquid solvents as microscopic viscous or solid bodies with nanosecond or slower dipole-relaxational dynamics [7,9, $12,13,23]$.

The analysis of molecular relaxation spectroscopy is based on the following considerations. Electronic excitation leads to a change of fluorophore dipole moment in magnitude and direction. Therefore, upon excitation the fluorophore initially is not in equilibrium with its dielectric environment. Equilibrium can be reached by relaxation of the electric field by rotation and displacement of the involved dipoles. This relaxation decreases the energy level of the excited state; in consequence the fluorescence spectra are shifted to longer wavelengths. Protein dynamics can be studied by observation of time dependent shifts of the fluorescence spectra as well as the time dependence of other spectroscopic effects of dipolar relaxation. If a system is described by a single dipolar relaxation time, $\tau_{\mathrm{R}}$, the shift of spectra with time should be exponential, and in principle the value of $\tau_{\mathrm{R}}$ can be determined [26]. In practice the determination is complicated by a non exponential emission decay, which was also found in the case of Na,K-ATPase (see Table 1). If there is a time-dependent shift with the rate comparable to the rate of emission, the emission is expected to decay faster at shorter wavelengths and slower at longer wavelengths; this has been observed in this study. Therefore, we could draw only the qualitative conclusion that $\tau_{\mathrm{R}}$ cannot be different substantially from $\tau_{F}$, and $\tau_{F}$ was determined to be about 5 ns.

The mean excited-state lifetime, $\tau_{\mathrm{F}}$, provides an intrinsic time marker that allows an analysis of the time-integrated steady state emission. Steadystate spectral shifts can be expressed as a function of $\tau_{F}$ and $\tau_{R}[38,26]$ :

$\frac{\bar{\nu}-\bar{\nu}_{\infty}}{\bar{\nu}_{0}-\bar{\nu}_{\infty}}=\frac{\tau_{\mathrm{R}}}{\tau_{\mathrm{F}}+\tau_{\mathrm{R}}}$

where $\nu$ is a wave number $(\nu=1 / \lambda)$ and $\bar{\nu}$ represents the position of the maximum of the spectrum. $\bar{\nu}_{0}$ is the wave number of the fluorescence maximum before relaxation, and $\bar{\nu}_{\infty}$ that of the fully relaxed state. To obtain $\bar{\nu}_{0}$ and $\bar{\nu}_{\infty}$ the temperature dependent properties of the system can be used. At the low temperature extreme the spectra represent the unrelaxed, and at the high temperature extreme the completely relaxed state. $\tau_{F}$ is assumed to be temperature independent and was taken to be 5 ns in our case. The experimental data (Fig. 2) can be reasonably approximated by a two-step relaxation mechanism. By applying eq. (1) the relaxation times, $\tau_{R}$, can be determined. Corresponding results are presented in Fig. 7. At $T_{1 / 2}^{0}=16^{\circ} \mathrm{C}$ we get from Fig. $2 \tau_{R}=\tau_{F}=5$ ns. Figure 7A demonstrates the dependence of $\tau_{\mathrm{R}}$ on temperature for the two ranges of relaxational shifts of fluorescence spectra, and Fig. 7B contains data in form of an Arrhenius plot $\left(\lg \tau_{\mathrm{R}}\right.$ vs. $\left.1 / T\right)$. Since we were not able to measure temperature-dependent life time data, we took this inconvenience into account by a systematic error, However, this error will not be significant, since the temperature-dependent variation of the life time is usually much smaller than the variations of the relaxation time. Estimated from changes in the fluorescence intensity, the average change in the life time is in our case 1.1 percent per degree. Since the slope of the curve corresponds to the activation energy of the dipole-relaxational mobility in the environ- 

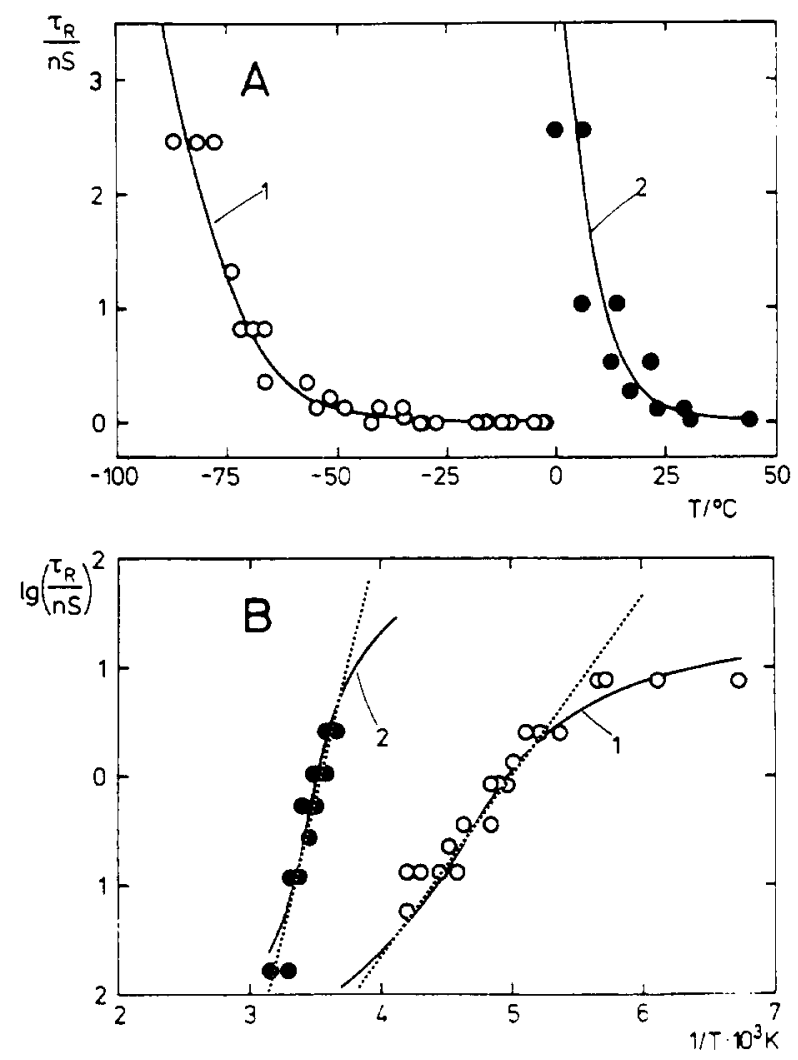

Fig. 7. (A) Results of determination of $\tau_{R}$ as a function of temperature for the lower (curve 1) and higher (curve 2) temperature interval of the maximum fluorescence wavelength shown in Fig. 2. (B): Presentation in Arrhenius coordinates.

ment of tryptophan residues, the latter presentation demonstrates that the activation energy is higher for the relaxations occurring in the higher temperature range.

The red-edge effect is associated with the distribution of the interaction energies between the protein and the environment due to the dynamics of the environment dipoles [7,9]. The dynamicsinduced rate, however, has to be slower or comparable to the rate of fluorescence emission. If the different micro-environments have relaxed before the emission occurs, no red-edge effect should be observed. If the magnitude of the rededge effect changes with temperature, the relaxation time of the environment is of the same time scale as the emission lifetime. $\tau_{\mathrm{R}}$ can be obtained from the equation $[7,9]$ :

$\frac{\nu-\nu^{\text {edge }}}{\nu_{0}-\nu_{0}^{\text {edge }}}=\frac{\tau_{\mathrm{R}}}{\tau_{\mathrm{F}}+\tau_{\mathrm{R}}}$ where $\nu-\nu^{\text {edge }}$ is the difference between the maxima of emission spectra excited at the mean and at the edge wavelength. $\nu_{0}-\nu_{0}^{\text {edge }}$ is the difference of a completely unrelaxed fluorophores. This condition can be realized at low temperatures. The dependencies of red-edge effect on temperature (Figs. 3 and 4) demonstrate the nanosecond rate of relaxations. Application of eq. (2) to ATPase of membrane fragments in $57 \%$ glycerol results in the values of $\tau_{\mathrm{R}}, 10 \mathrm{~ns}$ at $1^{\circ} \mathrm{C}, 6$ ns at $19^{\circ} \mathrm{C}$, and $3 \mathrm{~ns}$ at $35^{\circ} \mathrm{C}$ that confirm the results of time-resolved experiments at room temperatures.

It can be argued that the temperature-dependent shift may be caused by gradual static conformational changes in the protein rather than by relaxation. However, the following arguments are against this assumption. For all temperatures the maxima of the excitation-wavelength dependent fluorescence coincide at 305-307 nm (Figs. 3 and 4). This observation is a very characteristic feature of relaxation processes [7]. In the case of a conformational change, even if it is as small as shown in Fig. 6, the results should be different, one would expect that the spectrum is shifted at all wavelengths. In addition it is very unlikely that the same conformational change occurs in native membranes, in phospholipid vesicles, and under high concentrations of glycerol or sucrose. We could show recently that the activation energies of the enzymatic activity and the conformational changes are significantly different in native membranes and in reconstituted lipid vesicles (manuscript in preparation). If conformational fluctuations are contributing one could expect to observe different shifts in membrane and vesicle preparations.

\subsection{Fast dipole-relaxational dynamics and the function of $N a, K-A T P a s e$}

Kinetic studies of the $\mathrm{Na}, \mathrm{K}$ pump [4] suggest that the rate-limiting step of the pumping cycle under conditions close to physiological conditions is the $E_{1} \rightarrow E_{2}$ transition of the phosphorylated sodium form which controls occlusion/deocclusion of sodium ions and eventually the release of the first $\mathrm{Na}^{+}$[39]. At low ATP concentration 
the rate may be limited by the similar process of deocclusion of potassium ions which is controlled by $E_{2} \rightarrow E_{1}$ transition. This means that elementary steps of processes like ion binding, translocation and release should be fast events compared to the overall reaction rate. Therefore, it can be assumed, that the rates, in which dielectric relaxations of the protein matrix occur, are much faster than these elementary steps. When ions are bound to the protein molecule, they have to strip off at least part of their dehydration shell. The replacement of water molecules is accompanied by a rapid reorientation of polar groups within the protein matrix into equilibrium. Such dielectric stabilization compensates the high energy required for ion dehydration. Fast dielectric stabilization is probably involved also in reduction of repulsive forces between the three sodium or two potassium ions entering their binding sites and reduces the magnitude of activation energy barrier in their translocation.

A mechanism by which dielectric relaxation modifies energy barriers in an ion-transfer event has been studied in detail for ion permittivity and selectivity effects in ionic channels [15]. The energy profile for the ion was computed as a function of a distance along the channel including the term that describes the electrostatic interaction with the negative protein charges in the selectivity filter. To describe a time-dependent dielectric stabilization by protein dipoles, a Debye model of dielectric relaxations has been applied. It has been shown that the dielectric relaxations have a profound effect on the energy profile for the ion; this is accomplished by reducing the depths of the-energy wells in which the ions otherwise would be trapped. In this way the rate of ion transfer is controlled by the rate of dipolar relaxations. Probably this mechanism involving small-scale protein dynamics operates also for the $\mathrm{Na}, \mathrm{K}$ ATPase, and allows ion motions to be fast and not rate limiting.

The small difference of the fluorescence spectra between form $E_{1}$ and $E_{2}$ of the enzyme that we have found has been reported also by Chetverin and coworkers [31], who calculated difference spectra for both conformations. An explanation for the shift of the maximum indepen- dent of temperature and excitation wavelength has not been given so far. The shift of the fluorescence maximum may be caused by the conformational rearrangement between the form $\mathrm{Na}_{3} \mathrm{E}_{1}$ and the occluded form $E_{2}\left(K_{2}\right)$, which induces only small changes in the tryptophan environments. Another reason could be that the steric differences between $\mathrm{Na}^{+}$and $\mathrm{K}^{+}$ions cause a small difference of the polarization of the tryptophanes in the transmembrane segments which are close to the proposed ion binding moieties, while all other tryptophanes remain unaffected.

The fluorescence spectral characteristics presented here leads us to the conclusion, that the dynamics of the protein is fast in the nanosecond time scale. This dynamics reflects in some respect the elementary processes of the protein like transitions between minor conformational states or brownian movements. Ordered sequences of these fast elementary processes would aid the performance of the transitions between defined states in the pumping cycle of the Na,K-ATPase. Up to now we cannot discriminate between the importance of slower and faster relaxations on the functional abilities of the protein. Probably both are important for the transport properties of the sodium pump. They will be investigated in forthcoming experimental studies.

\section{Acknowledgements}

The authors wish to thank Dr. Robert Clegg, Göttingen, for support and critical discussion of the manuscript, Milena Roudna, Konstanz, and Sergey Skursky, Kiev, for excellent technical assistance. The work has been supported by the Deutsche Forschungsgemeinschaft (Sonderforschungsbereich 156).

\section{References}

1 L.C. Cantley, Curr. Top. Bioenerg. 11 (1981) 201.

2 I.M. Glynn, in: The enzymes of biological membranes, 2nd edn., vol. 3, Membrane transport, ed. A.N. Martonosi (Plenum Press, New York, 1985) p. 35.

3 A. Schwartz, R.J. Adams, W.J. Ball, J.H. Collins, S.S. Gupte, L.K. Lane, A.S. Reeves and E.T. Wallick, Int. J. Biochem. 12 (1980) 287. 
4 W. Stürmer, H.-J. Apell, I. Wuddel and P. Läuger, J. Membr. Biol. 110 (1989) 67.

5 R. Bühler, W. Stürmer, H.-J. Apell and P. Läuger, J. Membr. Biol. 121 (1991) 141.

6 W. Stürmer, R. Bühler, H.-J. Apell and P. Läuger, J. Membr. Biol. 121 (1991) 163.

7 A.P. Demchenko, Ultraviolet spectroscopy of proteins (Springer-Verlag, Berlin, 1986) p. 320.

8 D. Creed, Photochem. Photobiol. 39 (1984) 537.

9 A.P. Demchenko, Essays Biochem. 22 (1986) 120.

10 J.R. Lakowicz, Principles of fluorescence spectroscopy (Plenum Press, New York, 1983).

11 A.P. Demchenko, Tr. Biochem. Sci. 13 (1988) 374.

12 A.P. Demchenko, Eur. Biophys. J. 16 (1988) 122.

13 A.P. Demchenko and A.S. Ladokhin, Biochim. Biophys. Acta 955 (1988) 352.

14 A.P. Demchenko, I. Gryczynski, Z. Gryczynski, W. Wiczk, H. Malak and M. Fishman, Biophys. Chem. 48 (1993) 39.

15 Demchenko, N.N. Kositsky and V.I. Teslenko, Biophys. Chem. 35 (1990) 25.

16 A.P. Demchenko and V.A. Chinarov, J. Mol. Liq. 44 (1990) 189.

17 P.L. Jørgensen, Biochim. Biophys. Acta 356 (1974) 36.

18 P.L. Jørgensen, Biochim. Biophys. Acta 694 (1982) 27.

19 A. Schwartz, K. Nagano, M. Nakao, G.E. Lindenmayer and J.C. Allen, Meth. Pharmacol. 1 (1971) 361.

20 O.H. Lowry, J. Biol. Chem. 193 (1951) 265.

21 H.-J. Apell, M.M. Marcus, B. Anner, H. Oetliker and P. Läuger, J. Membr. Biol. 85 (1985) 49.

22 D.W. Piston, G. Marriott, T. Radivoyevich, R.M. Clegg, T.M. Jovin and E. Gratton, Rev. Sci. Instrum. 60 (1989) 2596.

23 K. Gekko and S.N. Timasheff, Biochemistry 20 (1981) 4667 .
24 G.C. Na and S.N. Timasheff J. Mol. Biol. 151 (1981) 165.

25 J.M. Beechem and L. Brand, Annu. Rev. Biochem. 54 (1985) 43.

26 N.G. Bakhshiev, Spectroscopy of intermolecular interactions, (Nauka publ., Leningrad, 1972) p. 263.

27 W.R. Ware, S.K. Lee, G.J. Brant and P. Snow, J. Chem. Phys. 54 (1971) 4729.

28 G.E. Shull, A. Schwartz and J.B. Lingrel, Nature 316 (1985) 691.

29 Yu.A. Ovchinnikov, N.N. Modyanov, N.E. Broude, K.E. Petrukhin, A.V. Grishin, N.M. Arzamazova, G.S. Monastyrskaya and E.D. Sverdlov, FEBS Lett. 201 (1986) 237.

30 N.N. Modjanov, S. Lutsenko, E. Chertova, R. Efremov and D. Gulyaev, Acta Physiol. Scand. 146 (1992) 49.

31 A.B. Chetverin, S.Y. Venyaminov, V.I. Emelyanenko and E.A. Burstein, Eur. J. Biochem. 108 (1980) 157.

32 E.A. Burstein, N.S. Vednkina and M.N. Ivkova, Photochem. Photobiol. 18 (1973) 263.

33 P.A. Tyson and M. Steinberg, J. Biol. Chem. 262 (1987) 4644.

34 N.A. Nemkovich, A.N. Rubinov and V.I. Tomin, in: Topics in fluorescence spectroscopy, ed. J.R. Lakowicz, vol. 2, (Plenum Press, New York, 1991) p. 367.

35 J. Griedrich and D. Harer, Angew. Chem. Int. Ed. Engl. 23 (1984) 113.

36 J.D. Simo, and Shyh-Gang Su, Chem. Phys. 152 (1991) 143.

37 C.F. Chapman, R.S. Fee and M. Maroncelli, J. Phys. Chem. 94 (1990) 4929.

38 Yu.T. Mazurenko and N.G. Bakhshiev, Opt. Spectrosk. (USSR) 28 (1970) 905.

39 W. Stürmer and H.-J. Apell, FEBS Lett. 300 (1992) 1.

40 A. Warshel and S.T. Russel, Q. Rev. Biophys. 17 (1984) 283. 